\title{
Molecular Diagnosis of Enteroviruses Associated with Hand, Foot and Mouth Disease (HFMD)
}

\author{
D. P. Sinha • C. G. Raut • H. Jayaprakash • \\ H. Hanumaiah • N. J. Shaikh • M. J. Manjunatha
}

Received: 13 February 2014 / Accepted: 10 May 2014 / Published online: 10 June 2014

(C) Dr. K C Chaudhuri Foundation 2014

To the Editor: Hand, Foot and Mouth Disease (HFMD) is a mild, self-limiting, exanthematous disease of infants and children below 10 y of age. Human enterovirus 71 (HEV-71) and Coxsackievirus A16 (CV-A16) are common etiological agents causing HFMD epidemics. HFMD usually spreads from person-to-person through contact with nose or throat discharges, feces or vesicular fluid [1].

The present study reports the investigation of enterovirus (EV) etiology in cases of HFMD that occurred during September through November 2013 in and around Bangalore, Karnataka. Seven suspected pediatric cases, who presented to the IGICH and Victoria Hospital, Bangalore, with clinical evidence of Hand, Foot and Mouth disease, were studied; the mean age was 2 and $5 \mathrm{y}$. Viral RNA was extracted from urine samples using the QiAmp viral RNA kit and viral RNA were tested by RT-PCR using enterovirus primers and HFMD associated specific CV-A16 primers [2]. Among the 7 cases, 4 cases were EV positive, out of which 2 cases were detected as serotype CV-A16 by RT-PCR.

In India, the role of enteroviruses in causing HFMD is not very clear on account of lack of accurate diagnosis, as most of the cases reported earlier have been diagnosed on the basis of clinical signs compatible with HFMD [3]. In the present study, use of sensitive molecular method, RT-PCR targeted against conserved and variable regions of the EV genome revealed the presence of significant EV RNA positivity [2].

D. P. Sinha $\cdot$ C. G. Raut $(\varangle) \cdot$ H. Jayaprakash $\cdot$ H. Hanumaiah $\cdot$ N. J. Shaikh • M. J. Manjunatha

National Institute of Virology, Bangalore Unit, Near NIMHANS, Karnataka 560 029, India

e-mail: cgraut@gmail.com
CV-A16 has attracted less attention probably due to its association often with mild and benign clinical symptoms. Therefore, there has been much less data about CA16 than EV71 [4]. But the HFMD cases reported here support the previously observed characteristics of CV-A16 infection [5].

A small outbreak of HFMD can become a major epidemic and because of genetic recombination of viruses, a benign illness can become a deadly disease as has happened in Asian countries [4]. It is important for dermatologists to be aware of this disease as they may be the first health care professionals to be consulted in such cases.

\section{Conflict of Interest None.}

Source of Funding The study is funded by National Institute of Virology, Pune.

\section{References}

1. Russo DH, Luchs A, Machado BC, Carmona Rde C, Timenetsky MC. Echovirus 4 associated to hand, foot and mouth disease. Rev Inst Med Trop Sao Paulo. 2006;48:197-9.

2. Gopalkrishna V, Patil PR, Patil GP, Chitambar SD. Circulation of multiple enterovirus serotypes causing hand, foot and mouth disease in India. J Med Microbiol. 2012;61:420-5.

3. Sarma N, Sarkar A, Mukherjee A, Ghosh A, Dhar S, Malakar R. Epidemic of hand, foot and mouth disease in West Bengal, India in August, 2007: a multicentric study. Indian J Dermatol. 2009;54:26-30.

4. Li Y, Zhu R, Qian Y, Deng J, Sun Y, Liu L, et al. Comparing Enterovirus 71 with Coxsackievirus A16 by analyzing nucleotide sequences and antigenicity of recombinant proteins of VP1s and VP4s. BMC Microbiol. 2011;11:246.

5. Saoji VA. Hand, foot and mouth disease in Nagpur. Indian J Dermatol Venereol Leprol. 2008;74:133-5. 